\title{
Off-design Considerations of the 50cc Penn State Ventricular Assist Device
}

\author{
Leslie A. Oley, Keefe B. Manning, Arnold A. Fontaine, and Steven Deutsch \\ Department of Bioengineering, The Pennsylvania State University, University Park, PA, U.S.A.
}

\begin{abstract}
Particle image velocimetry is used to study the flow field associated with off-design conditions of the 50cc Penn State Ventricular Assist Device. Phase average data, for 300 consecutive beats, was acquired for 60,75 , and 90 beats per minute (bpm) at 35/65 systolic duration and at $75 \mathrm{bpm}$ for 50/50 systolic duration. Diastolic flow is dominated by the inflow jet and shows more penetration and better wall washing at higher beat rates and shorter diastolic time. A flow separation near the minor orifice of the
\end{abstract}

aortic valve, however, is larger under these conditions. Turbulent energy, which peaks during diastole, is found to be strongest at the edges of the jet and near the valves and scales well with the maximum inlet jet velocity. A region of potential separation on the right lateral wall is identified and will be relieved by geometric modifications of the evolving design. Key Words: Assist device-Flow patterns-Thrombus deposition-Wall shear rates.
Penn State's 70cc pneumatic left ventricular assist device (LVAD) has been implanted clinically since 1985. These heart-assist pumps have seen success rates of over $90 \%$ and show promise in helping to reduce problems caused by limited donor heart availability and subsequent transplant opportunities. The recent REMATCH study (1) found that the LVAD is superior to drug therapy for those patients not eligible for heart transplants.

Despite this clinical success, there is a need for smaller devices to accommodate a wider range of potential recipients (2). Preliminary attempts to scale down the $70 \mathrm{cc}$ Penn State LVAD to a $15 \mathrm{cc}$ pediatric model, however, have proven unsuccessful, as a result of thrombus formation within the pumping chamber during animal implants (3). Unfavorable flow characteristics, particularly regions of low shear rate within the smaller chambers are the most likely cause of the thrombus formation (4-6). Evidence suggests that thrombus formation and deposition is predominantly a function of fluid shear stresses and blood residence times $(3,7-12)$ in these devices. In

Received November 2004; revised January 2005.

Address correspondence and reprint requests to Dr. Steven Deutsch, Department of Bioengineering, The Pennsylvania State University, 205 Hallowell Building, University Park, PA 16802, U.S.A. E-mail: sd1@wt.arl.psu.edu addition, the mechanical heart valves employed in the device can contribute to blood cell damage and activation through their elevated wall shear, regurgitant jets, and turbulence $(11,13)$. Brown et al. (7) have observed that the sequence of platelet damage and subsequent aggregation is extremely sensitive to shear stress. Research by Baldwin et al. (14) in a Penn State VAD has shown that the presence of a single rotational flow pattern during late diastolic filling helps to promote wall washing, which helps to minimize thrombus formation and deposition within the heart chamber. Hochareon et al. $(5,15)$ have shown that the formation and strength of this rotational flow are strongly dependent on both the sac motion and the early diastolic inlet jet.

A 50cc version of the Penn State LVAD entered the development phase in 1998, with a research goal of reducing thrombus formation in smaller devices. Hochareon et al. $(4,5)$ conducted the first investigation of the fluid dynamic patterns found in this $50 \mathrm{cc}$ LVAD under normal operating conditions ( 75 beats/ min [bpm]), using particle image velocimetry (PIV). Regions of flow stagnation and low wall shear stress were found to correlate to areas of thrombus deposition in animal implant studies $(6,16)$.

Flow patterns at off-design conditions, such as those encountered during lower or higher heart rates are of interest because the LVAD must often be run 
off-design to maintain flow rate. Jarvis et al. (17) have studied the dependence of formed blood element response on heart rate and systolic duration by pumping human blood through a mock circulatory loop using a 70cc Penn State Artificial Heart. Free hemoglobin, platelet number, and beta-thromboglobulin levels-indicators of the levels of hemolysis and platelet activation/damage-revealed that higher heart rates and longer systolic durations induced more hemolysis (17). Jarvis et al.'s (17) findings are consistent with the hypothesis that the altered fluid flow patterns within the heart chamber encountered at off-design conditions will have a direct effect on thrombus formation and deposition.

Here, we consider the impact of off-design beat rate and systolic duration on the flow field of a 50cc Penn State LVAD, using PIV. Data is presented and analyzed for heart rates of 60,75 , and $90 \mathrm{bpm}$ at $35 \%$ systolic duration and at $75 \mathrm{bpm}$ for $50 \%$ systolic duration.

\section{EXPERIMENTAL METHODS}

\section{0cc Penn State LVAD and mock circulatory flow loop}

The 50cc Penn State LVAD model, as shown in Fig. 1, was machined from Plexiglas to allow for optical access. Table 1 contains the dimensions of the major regions in the device. The model was fitted with $21 \mathrm{~mm}$ (aortic) and $23 \mathrm{~mm}$ (mitral) BjorkShiley (Shiley Inc., Irvine, CA, U.S.A.) monostrut valves with pyrolytic carbon discs to maintain unidirectional flow through the heart model. The mitral valve in this experiment was seated at zero degrees with respect to the horizontal axis, as illustrated in Fig. 2.

A polyurethane diaphragm was placed over a positive-displacement pusher plate. In a sac-driven or diaphragm LVAD, such as the model used in this experiment, the dynamics of the sac motion strongly influences the fluid flow patterns in the heart cham-

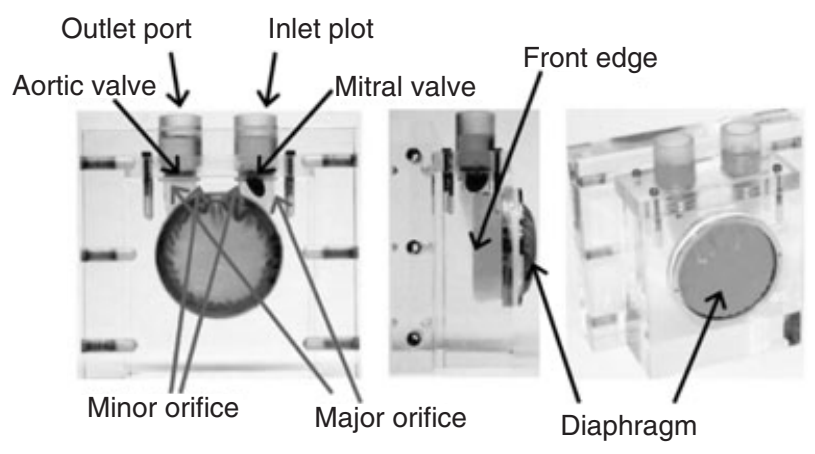

FIG. 1. 50cc Penn State LVAD acrylic chamber.
TABLE 1. $50 c c$ Penn State LVAD dimensions

\begin{tabular}{lc}
\hline Chamber location & Dimensions $(\mathrm{mm})$ \\
\hline Chamber radius & 34.3 \\
Mitral port diameter & 23 \\
Aortic port diameter & 21 \\
Chamber thickness & 18.8 \\
\hline
\end{tabular}

ber (15). Hochareon et al. (15) observed that having the diaphragm free of the pusher plate provided a better match to the clinical configuration, and that "free diaphragm" condition was maintained here.

The current experiments were performed with the 50cc LVAD pumping through a Penn State mock circulatory loop (18), driven by a Harvard positivedisplacement piston pump (Harvard Apparatus Co, Inc., Millis, MA, U.S.A.). The inlet and outlet flow waveforms were measured using two ultrasonic flow meters (Transonic Systems Inc., Ithaca, NY, U.S.A.). The pusher plate displacement, and hence stroke length, was measured by a linear velocity displacement transducer (Solartron Metrology, Bognor Regis, U.K.). Two pressure transducers, located within the inlet and outlet compliance chambers, were used to monitor the inlet and outlet pressure waveforms (Maxxim Medical, Athens, TX, U.S.A.).

The blood analog fluid used in the current study was a mineral oil, with a dynamic viscosity of approximately $4.6 \mathrm{cP}$ and a density of $0.83 \mathrm{~g} / \mathrm{cc}$ at $25^{\circ} \mathrm{C}$. The refractive index of the mineral oil was 1.46 , which closely matched that of the Plexiglas model (1.488) and thereby reduced image distortions caused by refraction at the model-fluid interface. The blood analog fluid was seeded with $10 \mu \mathrm{m}$ hollow glass beads (Potters Industries Inc., Valley Forge, PA, U.S.A.). The particle density (particles/volume of fluid) was regulated to provide adequate seeding density (usually defined as at least 5 particle pairs per PIV interrogation region (19)).

For the comparative study of fluid flow characteristics at off-design heart rates, three different beat

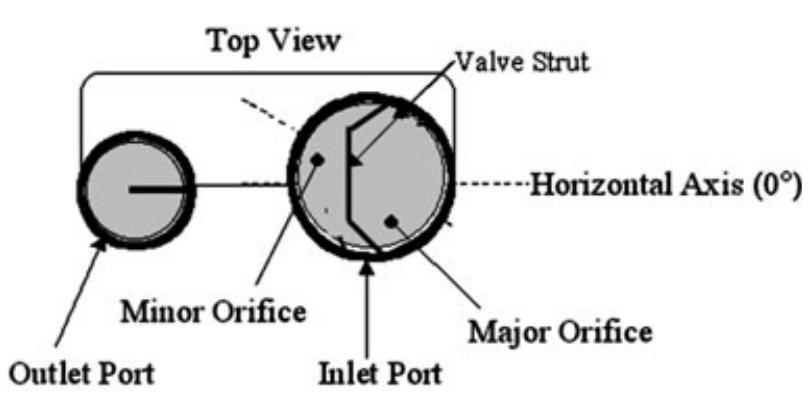

FIG. 2. Mechanical heart valve orientations within the LVAD chamber. 
rates were considered: 75,90 , and $60 \mathrm{bpm}$. As the focus of this study was to assess the effect of beat rate, the cardiac output and aortic and atrial pressure and flow waveforms were kept reasonably constant. To maintain these parameters, the stroke length, systemic resistance, systemic compliance, and atrial head were altered accordingly. The systolic duration for these tests was chosen to be approximately $35 \%$ of the cycle. The aortic (outlet) pressure was adjusted to closely reflect "normal" operating conditions of $120 / 80 \mathrm{~mm} \mathrm{Hg}$ (14). The atrial filling pressure was maintained at approximately $30 / 5 \mathrm{~mm} \mathrm{Hg}$. The inlet flow rate was maintained at an average of approximately $4.8 \mathrm{~L} / \mathrm{min}$, with a peak atrial flow rate between 16 and $18 \mathrm{~L} / \mathrm{min}$. To compare the effect of systolic duration on fluid flow characteristics within the 50cc LVAD chamber, a data set was acquired at $75 \mathrm{bpm}$ with a systolic duration of $50 \%$. Again, the cardiac output and pressure waveforms were comparable.

\section{Particle image velocimetry}

Particle image velocimetry (PIV) was used as the optical measurement technique to produce global views of instantaneous flow fields within the $50 \mathrm{cc}$ Penn State LVAD chamber. The light sources for the PIV experiments were dual pulsed Nd:YAG lasers (New Wave Research Inc., Fremont, CA, U.S.A.) with approximately $120 \mathrm{~mJ} /$ pulse at $532 \mathrm{~nm}$ wavelength, with a pulse width of about $10 \mathrm{~ns}$. Both lasers were fired at a Q-switch delay corresponding to the high energy region of the energy curve, producing an approximate $6 \mathrm{~mm}$ diameter beam with both good spatial uniformity and pulse-to-pulse repeatability. The time separation of the two laser pulses must be small enough to minimize particle loss between the first and second frames of the interrogation window (19). However, the time separation must be long enough to permit adequate displacement of particles at the lowest measurable velocities in each velocity component and to minimize the impact of pixel peak locking (20).

The PIV images of the flow field within the LVAD were captured using a PIVCAM 10-30 CCD camera (TSI, Minneapolis, MN, U.S.A.), which takes two images per capture. The CCD camera has a $1000 \times 1016$ pixel CCD chip with a $9 \mu \mathrm{m} /$ pixel square spacing, and was operated at a fixed frame rate of $30 \mathrm{~Hz}$, with a shutter open time of $225 \mu$ s. Physical resolution is approximately $85 \mu \mathrm{m} /$ pixel.

Our measurements were all acquired at a plane $5 \mathrm{~mm}$ from the front wall-a location with flow patterns typical of the central region of the pump. A $25 \mathrm{~mm}$ diameter high-energy mirror, coupled with a
$25 \mathrm{~mm}$ cylindrical lens and $500 \mathrm{~mm}$ spherical lens system, was used to create a laser sheet that was $20 \mathrm{~cm}$ wide and approximately $0.5 \mathrm{~mm}$ thick.

The intensity of the laser sheet is attenuated as it travels through the blood analog fluid. In addition, optical access may be compromised at certain points during the cardiac cycle as a result of interference caused by the pusher plate and diaphragm. Since the fluid flow characteristics during filling are most important in these devices $(14,15)$, the light sheet optics were placed on the inlet side of the model.

The pulsatile flow field characteristics within the heart chamber were studied by phase window averaging the velocity data at 10 phases of the cardiac cycle: five are reported in this study $(12.5 \%, 18.75 \%$, $25 \%, 37.5 \%, 75 \%)$. The effective phase window width was always less than $0.5 \mathrm{~ms}$. Initial data were acquired at the "normal" operating conditions of $75 \mathrm{bpm}$ and $35 \%$ systolic duration. The TSI LaserPulse Synchronizer was externally triggered off the atrial flow waveform, to capture one image per cardiac cycle at each phase of interest. A sequence of 300 images was acquired at each phase of the cardiac cycle, using consecutive beats. The laser pulse separation and pulse delay values were adjusted to optimize image acquisition and particle displacement for a given flow field prior to collection of each 300 image sequence. For the comparative study between different heart rates, the 10 time frames were scaled as a percentage of the cycle. For example, the flow fields are compared at $18.75 \%$ of the cardiac cycle from the start of diastole for 60,75 , and $90 \mathrm{bpm}$.

The phase-window-acquired data were processed using Insight 5 software (TSI, Minneapolis, MN, U.S.A.). The software segments the images into small "interrogation regions" and computes the average particle displacement within a region by performing a spatial cross-correlation between the two consecutive PIV images. The final dimensions of the interrogation regions were chosen to minimize noise and increase the accuracy of the planar velocity estimation. Smaller interrogation regions provide better spatial resolution of the measured velocity field, but increase the chance for in-plane particle loss and put added constraints on seeding density (19). A recursive Nyquist grid was used with the final interrogation region set to a $16 \times 16$ pixel size. This gave approximately 15 particle pairs in each $16 \times 16$ pixel interrogation region. The cross-correlation algorithm may be thought of as pattern recognition of the particle distribution between the two captured images within an interrogation region (19). A crosscorrelation peak is found and represents the average particle displacement within the interrogation region 
(19). The Hart Correlation, which is a crosscorrelation-based correction algorithm with sparse array compressed image correlation, was used with the bilinear peak engine (21).

\section{EXPERIMENTAL RESULTS AND DISCUSSION}

\section{Heart rate comparison}

Phase averaged maps at $12.5 \%$ after the onset of diastole, just before the peak inflow, are shown in Fig. 3. The peak inlet jet velocity becomes progressively faster as the heart rate increases: $60 \mathrm{bpm}$ (Fig. 3a) has maximum velocities of approximately $1.8 \mathrm{~m} / \mathrm{s}, 75 \mathrm{bpm}$ (Fig. 3b) has maximum velocities of approximately $2.04 \mathrm{~m} / \mathrm{s}$, and $90 \mathrm{bpm}$ (Fig. 3c) has maximum velocities of approximately $2.14 \mathrm{~m} / \mathrm{s}$. This increase is generally consistent as the heart is pumping similar average flow rates, but at relatively shorter diastolic times as heart rate increases. The increase observed, however, is considerably less than one would expect $(1: 1.25: 1.5)$ if the diastolic duration were the only parameter, so that the characteristic size of the inflow jet must also increase with increased beat rate. It is apparent that as the heart rate increases, the inlet jet strengthens and penetrates further towards the bottom of the chamber. A higher wall shear stress is anticipated, on the same order as the velocity change, at the bottom of the chamber with increasing heart rate. At a heart rate of $90 \mathrm{bpm}$, the beginning of the diastolic rotational flow is clear. Additionally, at the higher heart rates (75 and $90 \mathrm{bpm}$ ), the inlet jet hugs the right lateral wall further into the heart chamber, which may decrease the potential for flow separation there. These characteristics at the elevated heart rate may be desirable to deter thrombus formation and deposition in smaller-sized positive displacement LVADs.

In Fig. 4 near peak diastole, $18.75 \%$ after the start of inflow, the inlet jets have spread further down into the chamber. The peak inlet velocities are roughly the same magnitude as at the previous phase, between 1.8 and $2.15 \mathrm{~m} / \mathrm{s}$, with faster maximum velocities corresponding to faster heart rates. The inlet jet at $90 \mathrm{bpm}$ (Fig. 4c) appears to hug the right lateral wall closer than the jets at the other two heart rates. The area of the possible flow separation along the right and bottom wall of the heart chamber at 60 and $75 \mathrm{bpm}$ is greatly reduced at a heart rate of $90 \mathrm{bpm}$. The large-scale, rotational flow, characteristic of late diastole, is beginning to form for all beat rates at this time frame. Comparison of the figures show that at $90 \mathrm{bpm}$, the bottom, right lateral, and top walls of the chamber are experiencing higher fluid velocities than at the other heart rates.

In Fig. 5, at a time frame 25\% into the cycle, the inlet jets still maintain peak velocities above $1.7 \mathrm{~m} / \mathrm{s}$ in all cases. The rotational flow is becoming very prominent at this point. It is again apparent that the inlet jet at $90 \mathrm{bpm}$ penetrates further into the bottom of the chamber, with maximum velocities at this location of approximately $1.3 \mathrm{~m} / \mathrm{s}$. There are still areas of possible flow separation at the right lateral wall in all three velocity maps.

In the phase-averaged velocity vector maps, Fig. 6 , taken at late diastole, approximately $37.5 \%$ into the cardiac cycle, the central rotational flow is the most notable fluid dynamic pattern for all three heart rates. At $90 \mathrm{bpm}$, the highest near-wall velocities are found along the lateral and bottom portions of the chamber. These velocities are as high as $1.3 \mathrm{~m} / \mathrm{s}$, or about 1.8 times higher than those seen along the lateral and bottom portions of the chamber at a heart rate of $75 \mathrm{bpm}$. There is, however, a location of low flow (wall shear) at the top wall at a heart rate of $90 \mathrm{bpm}$ that is not seen at the other two heart rates.

Just before peak systole, Fig. 7, the aortic valve is fully opened, and the peak outflow is approximately $1.3 \mathrm{~m} / \mathrm{s}$ for each heart rate case. Each outlet jet appears to hug the lateral wall of the outlet port, especially in the case of $90 \mathrm{bpm}$. This may be due to the relatively short period of systolic ejection at the higher heart rate. At $90 \mathrm{bpm}$, there appears to be more top wall washing, as this portion of the chamber sees relatively high velocities (as high as $0.76 \mathrm{~m} / \mathrm{s}$ in some places) during the systolic ejection phase. This may improve wall washing in this area. There is, however, an apparent flow separation near the minor orifice wall of the aortic valve as was first shown by Baldwin et al. (14). The separated region appears to grow in size with increasing beat rate and is probably a consequence of the diastolic rotational motionwhich is in the opposite direction to that of the systolic flow. Higher velocities near the top wall, associated with the higher beat rates, would be harder to turn toward the inlet valve and therefore more prone to separation. In general, however, clot formation has not been observed at the top wall and valve areas.

\section{Systolic duration comparison}

The systolic duration study is a comparative investigation between the previously displayed data at $75 \mathrm{bpm}$ with a $35 \%$ systolic duration and data acquired at $75 \mathrm{bpm}$ with $50 \%$ systolic duration. As the $50 \%$ systolic duration has a shorter diastolic time (400 $\mathrm{ms}$ to $520 \mathrm{~ms}$ ), higher speed inlet jets are anticipated and more penetration for that case and, in 
(a)

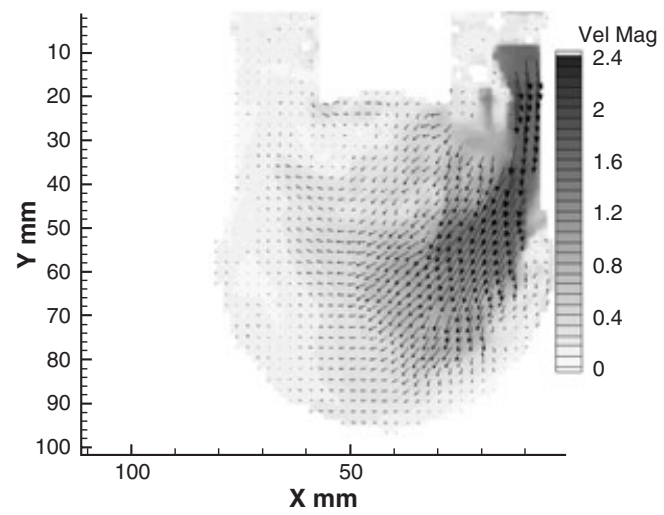

(b)

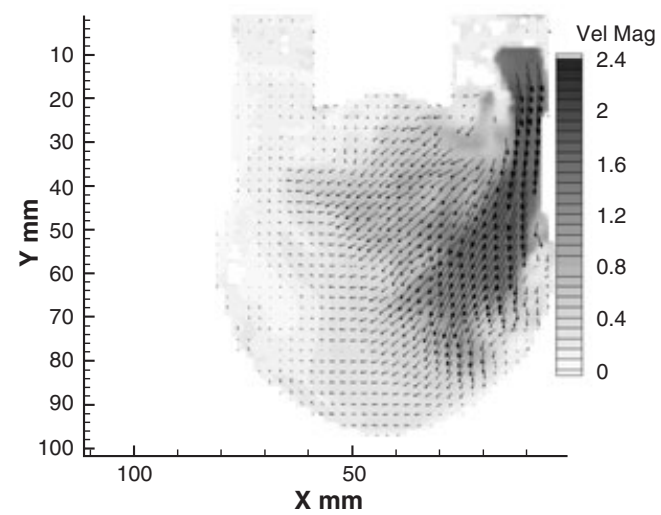

(c)

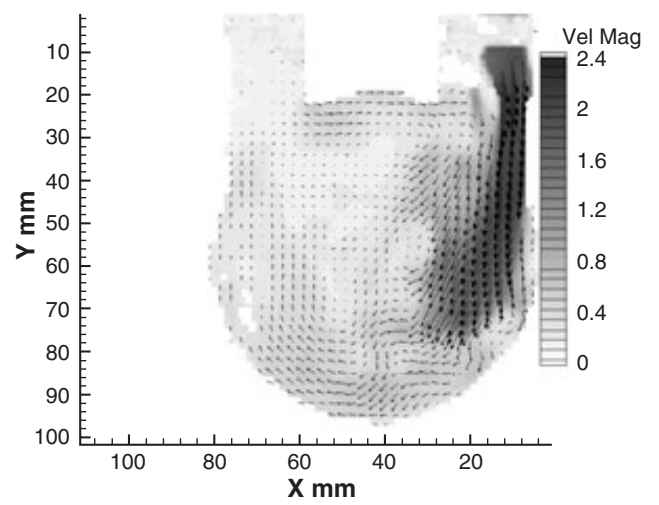

FIG. 3. $50 \mathrm{cc}$ PIV data at $12.5 \%$ into the cardiac cycle after the onset of diastole: (a) $60 \mathrm{bpm}$, (b) $75 \mathrm{bpm}$, and (c) $90 \mathrm{bpm}$. The systolic duration is $35 \%$. The strengths of the diastolic jets are compared.

general, this is observed. Typical results are shown in Fig. 4(d), near peak diastole at $18.75 \%$ into the cycle. Although the peak inlet velocities are similar, at about $2.1 \mathrm{~m} / \mathrm{s}$, clearly, the inlet jet for the $50 \%$ condition penetrates further-nearly to the bottom wall of the chamber. Once again, the diastolic rotational pattern is formed earlier for the $50 \%$ condition, promoting wall washing at the top of the model. As noted here, however, that inlet jet appears to be
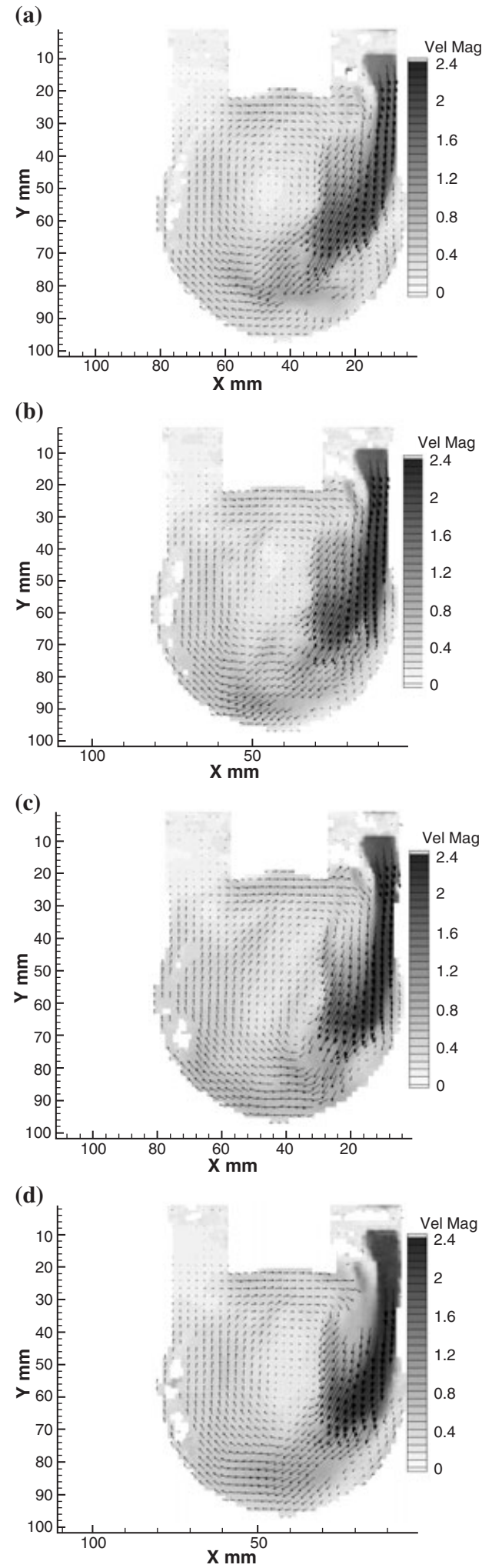

FIG. 4. $50 \mathrm{cc}$ PIV data at $18.75 \%$ into the cardiac cycle after the onset of diastole: (a) $60 \mathrm{bpm}$, (b) $75 \mathrm{bpm}$, (c) $90 \mathrm{bpm}$, all at 35\% systolic duration, and (d) $75 \mathrm{bpm}$ at $50 \%$ systolic duration. The regions of slow or separated flow on the right lateral wall are compared. 
(a)

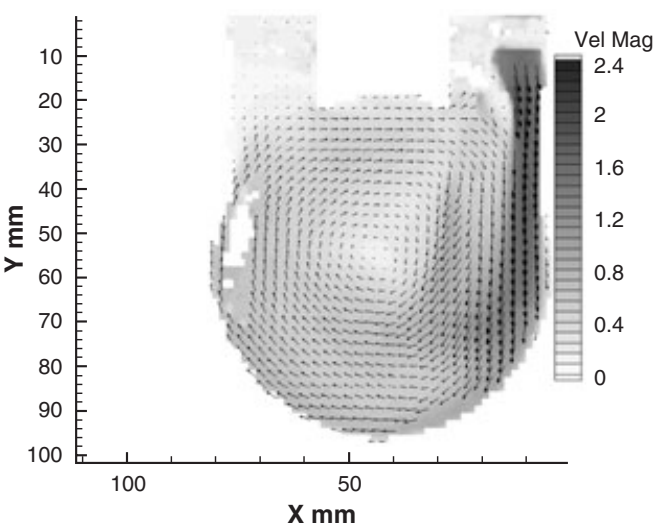

(b)

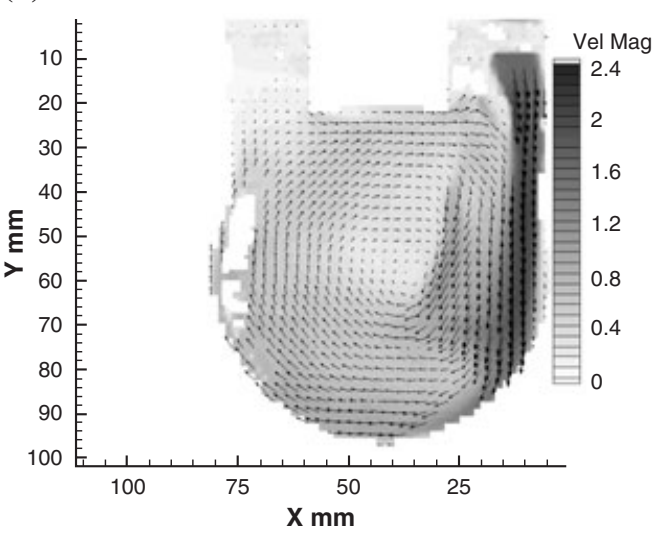

(c)

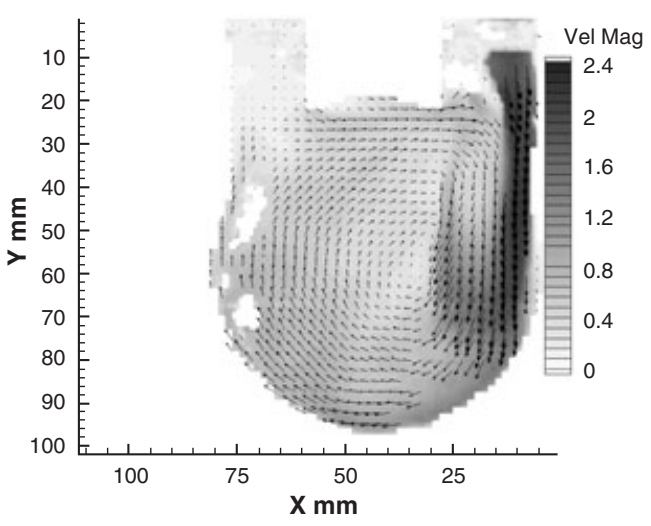

FIG. 5. $50 \mathrm{cc}$ PIV data at $25 \%$ into the cardiac cycle after the onset of diastole: (a) $60 \mathrm{bpm}$, (b) $75 \mathrm{bpm}$, and (c) $90 \mathrm{bpm}$. The systolic duration is $35 \%$. The patterns of rotational flow are compared.

more nearly directed toward the center of the chamber than does the jet at $35 \%$ systolic duration. This increases the region of slow flow near the right wall and may increase the potential for separation there.

\section{Turbulence energy}

In Fig. 8, the normalized turbulence energy at $18.75 \%$ after the onset of diastole for each of the beat rates at $35 \%$ systolic duration and for $75 \mathrm{bpm}$ at $50 \%$ systolic duration are shown. These results are quite typical of what is observed throughout early diastole and represent the largest values of turbulence intensity. In each case, the maximum inlet jet velocity squared was used as a normalization parameter, as this inlet jet is the most significant feature of the flow.
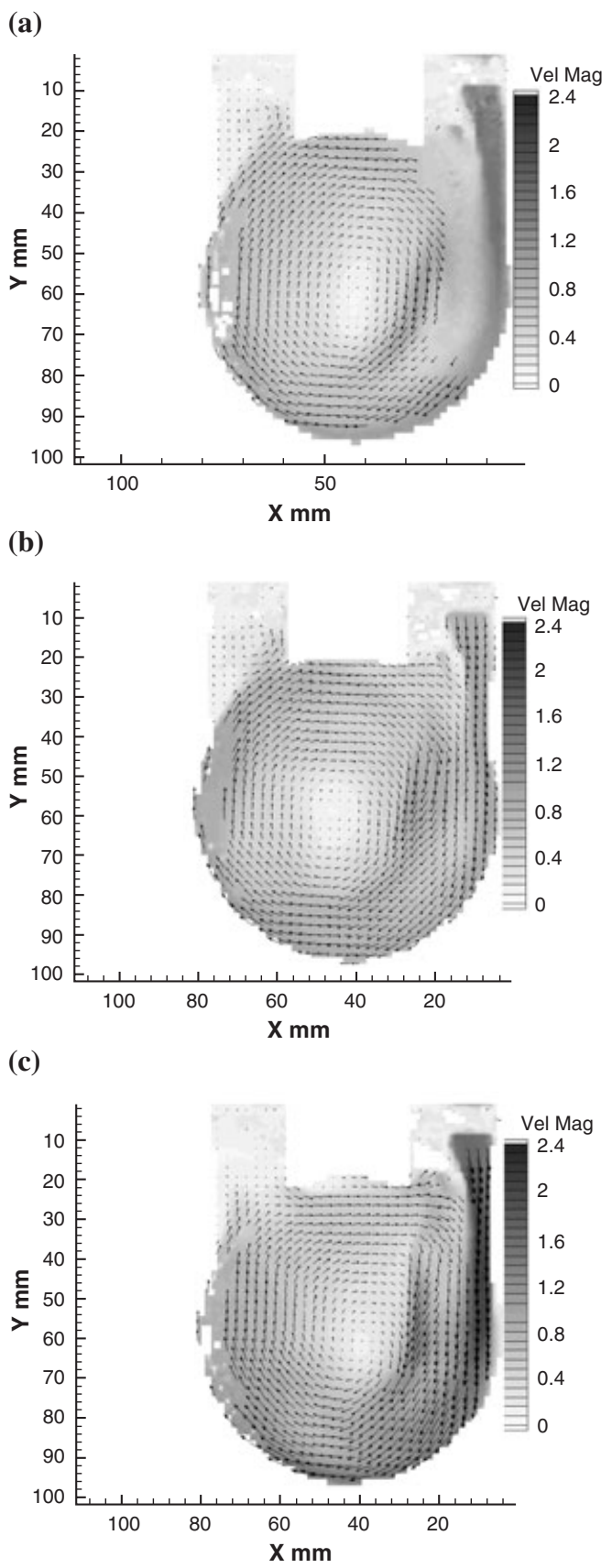

FIG. 6. $50 \mathrm{cc}$ PIV data at $37.5 \%$ into the cardiac cycle after the onset of diastole: (a) $60 \mathrm{bpm}$, (b) $75 \mathrm{bpm}$, and (c) $90 \mathrm{bpm}$. The systolic duration is $35 \%$. The flow patterns of the top wall are compared. 
(a)

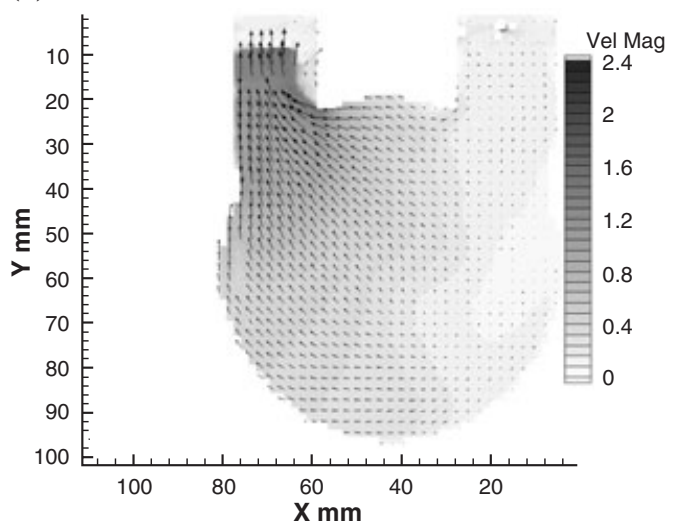

(b)

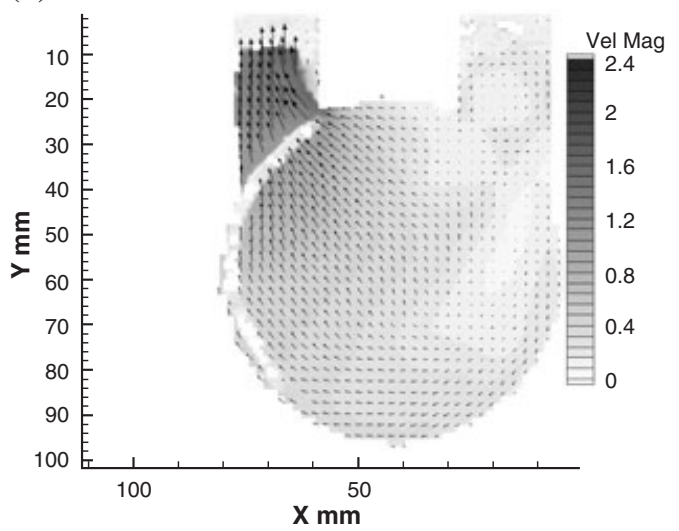

(c)

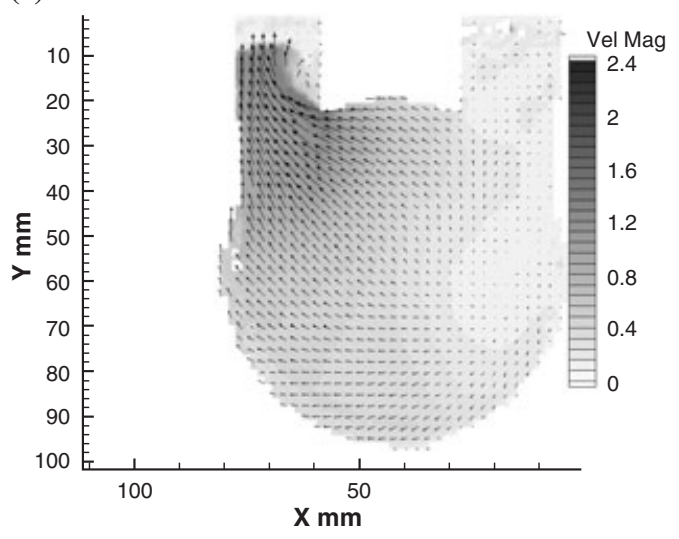

FIG. 7. 50cc PIV data at $75 \%$ into the cardiac cycle after the onset of diastole: (a) $60 \mathrm{bpm}$, (b) $75 \mathrm{bpm}$, and (c) $90 \mathrm{bpm}$. The systolic duration is $35 \%$. The regions of separation near the wall of the valve minor orifice are compared.

Certainly, some of the "energy" in each of the figures is contributed by beat-to-beat variation of the flow. In making comparisons, the assumption that this beat-to-beat variation is not a strong function of the beat rate. The results are plausible. The energy, for each case, is concentrated near the inlet valve and in the edges of the inlet jet during the onset of diastole. There is not a significant change in the pattern with (a)

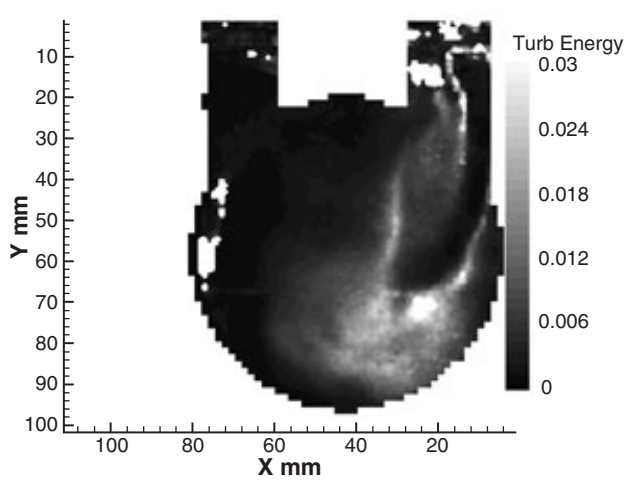

(b)

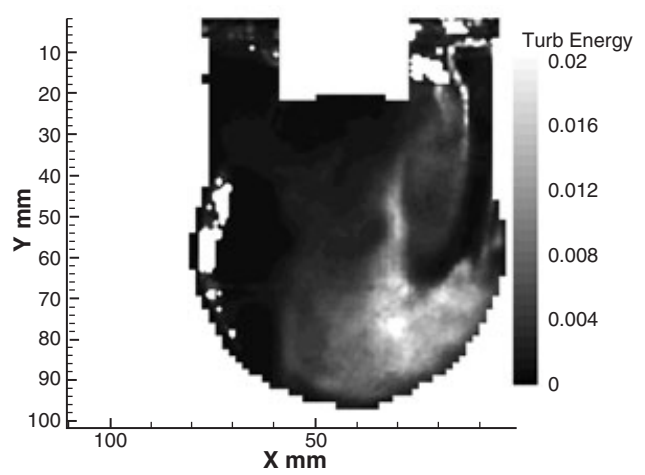

(c)

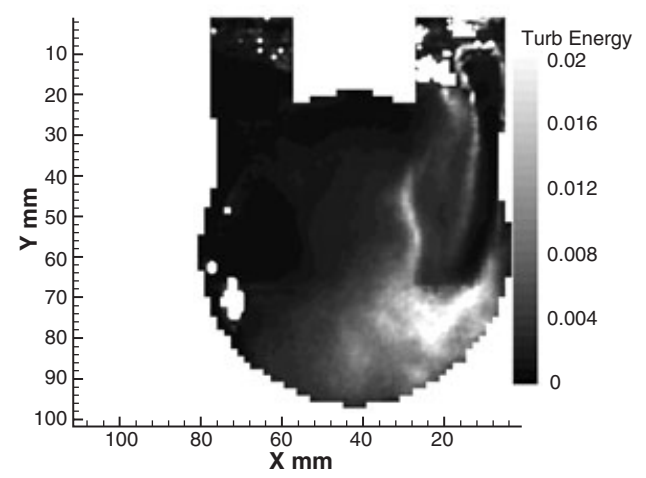

(d)

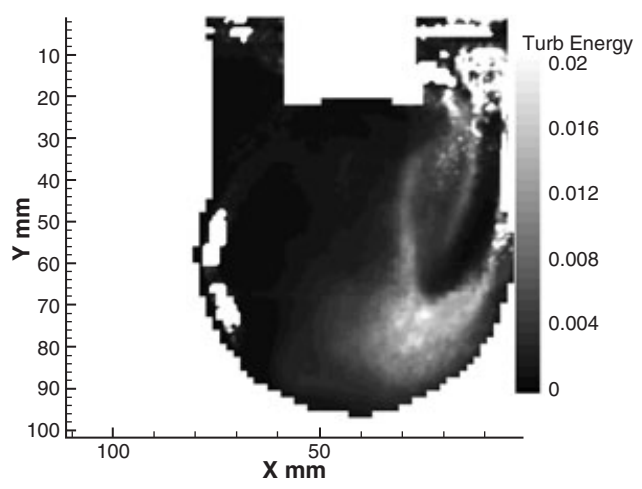

FIG. 8. Turbulence energy at $18.75 \%$ into the cardiac cycle after the onset of diastole: (a) $60 \mathrm{bpm}$, (b) $75 \mathrm{bpm}$, (c) $90 \mathrm{bpm}$, and (d) $75 \mathrm{bpm}$ (50\% systolic duration). Typical turbulence energy distributions for early diastole are compared. 
the changing parameters, and for each case the maximum value of the inlet jet appears to be a reasonable scaling parameter.

\section{CONCLUSIONS}

Thrombus formation and deposition remains a clinical problem with small artificial blood pumps and is thought to arise from altered internal flow dynamics resulting from size reduction. A pump's potential for hematological complications during implantation appears to increase as it is scaled down, as observed in in vivo studies of the current $50 \mathrm{cc}$ design and earlier pediatric designs $(3,16)$.

Particle image velocimetry was employed as a noninvasive optical measurement technique to quantify the fluid flow patterns within the 50cc LVAD at various times during the cycle. To maintain cardiac output, it is often necessary to run the LVAD at offdesign conditions, such as different heart rates. Since inefficient filling and poor wall washing can be problems with these devices, underlying fluid flow patterns during the diastolic phase are of particular concern.

In the current study, higher heart rates $(90 \mathrm{bpm})$ yielded higher velocities and likely higher wall shear rates along the bottom and lateral walls of the chamber during diastolic filling. Increased wall shear in these areas is clinically desirable, since these locations have shown thrombus deposition (16). As the inlet jet accelerates into the chamber through the mitral valve near peak diastole, it exhibits higher inlet velocities and penetrates further towards the bottom of the chamber at $90 \mathrm{bpm}$. In addition, near peak systole, there is increased washing along the top wall of the chamber, a region that may experience low flow velocities or flow stasis during filling at $90 \mathrm{bpm}$. Increased wall washing at the top of the chamber, however, appears associated with a larger separation region near the minor orifice of the aortic valve.

The current study also included an investigation into the effect of different systolic durations on fluid dynamic properties in the 50cc LVAD. In general, increasing the systolic duration is quite similar to increasing the beat rate as both decrease the diastolic fill time. We find, in addition, that the inflow jet is directed more toward the bottom center of the chamber for the longer systolic duration, increasing the risk of separation on the right lateral wall.

Mixing, as represented by the turbulence intensity, was not very sensitive to beat rate or systolic duration. The maximum velocity of the inlet jet, the dominant feature of diastole, was found to be an adequate velocity scale.
The right lateral wall appears throughout this study to be a region of low flow and potential separation. Changes in the design of the device, currently underway, will involve geometric modification of this region. This illustrates the value of PIV measurements as part of the design process. Although existing techniques, such as similarity, may be useful in guiding the overall design, it is the local fluid mechanics that will largely govern the thromboembolic state of the device. Particle image velocimetry seems uniquely capable of providing useful information about the local flow fields rapidly and accurately. The incorporation of PIV-based wall shear stress estimates, first introduced by Hochareon et al. (4) would be of additional benefit.

Finally, since the mechanical heart valves used with the LVAD play a central role in blood element damage, as well as filling characteristics of the chamber, changing the type (bileaflet or tilting disk) of valve and/or the valve orientation could prove to be clinically beneficial. It is clear from the current measurements, for example, that the Bjork-Shiley valves used are not optimal. Observations have yielded both a very weak minor orifice inlet flow and a nearly completely blocked minor orifice outlet flow, with separation near the minor orifice wall. It is clear that, for maximum benefit, not only must the best valves be selected but must in some way tailor the flow into these valves to improve their performance. This implies, of course, that the proper design of an LVAD will involve chamber, valve, and connector design combined.

Acknowledgments: Supported by NIH Grant HL60276. We also gratefully acknowledge Thea McSweeney for her assistance with the TecPlot figures.

\section{REFERENCES}

1. Rose EA, Moskowitz AJ, Packer M, et al. The REMATCH trial: rationale, design, and end points. Randomized Evaluation of Mechanical Assistance for the Treatment of Congestive Heart Failure. Ann Thorac Surg 1999;67:723-30.

2. Mehta SM, Weiss WJ, Snyder AJ, et al. Testing of a $50 \mathrm{cc}$ stroke volume completely implantable artificial heart: expanding chronic mechanical circulatory support to women, adolescents, and small stature men. ASAIO J 2000;46:779-82.

3. Bachmann C, Hugo G, Rosenberg G, Deutsch S, Fontaine A, Tarbell JM. Fluid dynamics of a pediatric ventricular assist device. Artif Organs 2000;24:362-72.

4. Hochareon P, Manning KB, Fontaine AA, Tarbell JM, Deutsch S. Wall shear-rate estimation within the 50cc Penn State Artificial Heart using particle image velocimetry. J Biomech Eng 2004;126:430-7.

5. Hochareon P, Manning KB, Fontaine AA, Tarbell JM, Deutsch S. A Fluid dynamic analysis of the $50 \mathrm{cc}$ penn state artificial heart under physiological operating conditions using particle image velocimetry. J Biomech Eng 2004;126:585-93. 
6. Hochareon P, Manning KB, Fontaine AA, Tarbell JM, Deutsch S. Correlation of in vivo clot deposition with the flow characteristics in the 50cc Penn State Artificial Heart: a preliminary study. ASAIO J 2004;50:537-42.

7. Brown CH, Leverett LB, Lewis CW, Alfrey CP, Hellums JD. Morphological, biochemical, and functional changes in human platelets subjected to shear stress. J Lab Clin Med 1975;86: $462-71$.

8. Sutera SP, Mehrjardi MH. Deformation and fragmentation of human red blood cells in turbulent shear flow. Biophys $J$ 1975;15:1-10.

9. Sallam AM, Hwang NH. Human red blood cell hemolysis in a turbulent shear flow: contribution of Reynolds shear stresses. Biorheology 1984;21:783-97.

10. Francischelli DE, Tarbell JM, Geselowitz DB. Local blood residence times in the Penn State artificial heart. Artif Organs 1991;15:218-24.

11. Yoganathan AP, Reul H, Black MM. Heart valve replacements: problems and developments. In: Hastings, GW, ed. Cardiovascular Biomaterials. Berlin: Springer-Verlag, 1992; 173-83.

12. Konig CS, Clark C. Flow mixing and fluid residence times in a model of a ventricular assist device. Med Eng Phys 2001;23:99-110.
13. Lu PC, Lai HC, Liu JS. A reevaluation and discussion on the threshold limit for hemolysis in a turbulent shear flow. J Biomech 2001;34:1361-4.

14. Baldwin JT, Deutsch S, Geselowitz DB, Tarbell JM. LDA measurements of mean velocity and Reynolds stress fields within an artificial heart ventricle. J Biomech Eng 1994;116: 190-200.

15. Hochareon P, Manning KB, Fontaine A, Deutsch S, Tarbell JM. Diaphragm motion affects flow patterns in an artificial heart. Artif Organs 2003;27:1102-7.

16. Yamanaka H, Rosenberg G, Weiss WJ, et al. A multiscale surface evaluation of thrombosis in left ventricular assist systems. ASAIO J 2003;49:222.

17. Jarvis P, Tarbell JM, Frangos JA. An in vitro evaluation of an artificial heart. ASAIO Trans 1991;37:27-32.

18. Rosenberg G. A mock circulatory system for in vitro studies of artificial hearts. PhD Thesis. University Park, PA: The Pennsylvania State University, 1972.

19. Raffel M, Willert CE, Kompenhans J. Particle Image Velocimetry: a Practical Guide. Berlin: Springer-Verlag, 1998.

20. Christensen KT. The influence of peak-locking errors on turbulence statistics computed from PIV ensembles. Exp Fluids 2004;36:484-97.

21. Hart DP. High-speed PIV analysis using compressed image correlation. J Fluids Eng 1998;120:463-70. 